Revue d'histoire de l'Amérique française

\title{
Diagnostiquer la " schizophrénie spirituelle » du Canada français : la politique spirituelle de Claude Ryan, 1952-1958
}

\section{Michael Gauvreau}

Volume 67, numéro 3-4, hiver-printemps 2014

URI : https://id.erudit.org/iderudit/1030039ar

DOI : https://doi.org/10.7202/1030039ar

Aller au sommaire du numéro

\section{Éditeur(s)}

Institut d'histoire de l'Amérique française

\section{ISSN}

0035-2357 (imprimé)

1492-1383 (numérique)

Découvrir la revue

\section{Citer cet article}

Gauvreau, M. (2014). Diagnostiquer la « schizophrénie spirituelle » du Canada français : la politique spirituelle de Claude Ryan, 1952-1958. Revue d'histoire de l'Amérique française, 67(3-4), 375-401. https://doi.org/10.7202/1030039ar
Résumé de l'article

Cet article analyse le rôle intellectuel de Claude Ryan dans une transformation culturelle de grande envergure au sein de l'Action catholique au Québec de 1945 à 1958. Durant ces années, la pensée de Ryan fut fortement influencée par de nouveaux courants de théologie catholique et la psychologie sociale dominant l'horizon de nombreux intellectuels américains. Sous son inspiration, l'Action catholique abandonne progressivement sa préoccupation initiale avec la réforme sociale, dominée par la « question des travailleurs » et la notion de la jeunesse comme classe à part dans la société moderne, pour une inquiétude centrée sur la classe moyenne québécoise, une orientation plus psychologique, centrée sur l'existence des mentalités religieuses et culturelles conflictuelles. Dès le milieu des années 1950, Ryan décerna dans ce conflit un vecteur dynamique de la sécularisation, mais il demeura plutôt optimiste que ces contemporains trouveraient, par le biais d'un catholicisme plus centré sur le spirituel, les ressources culturelles pour surmonter ce qu'il identifiait comme la " crise de l'adolescence » du Canada français.
Tous droits réservés @ Institut d’histoire de l'Amérique française, 2015

Ce document est protégé par la loi sur le droit d'auteur. L'utilisation des services d'Érudit (y compris la reproduction) est assujettie à sa politique d'utilisation que vous pouvez consulter en ligne.

https://apropos.erudit.org/fr/usagers/politique-dutilisation/ 


\title{
Diagnostiquer la "schizophrénie spirituelle " du Canada français ${ }^{\mathrm{I}}$ : la politique spirituelle de Claude Ryan, I952-1958
}

\author{
Michael Gauvreau \\ Department of History \\ McMaster University
}

«L'inimitié qui existait naguère entre le monde et l'Église, est maintenant transportée au cœur de l'Église elle-même²»

RÉsumÉ - Cet article analyse le rôle intellectuel de Claude Ryan dans une transformation culturelle de grande envergure au sein de l'Action catholique au Québec de 1945 à 1958. Durant ces années, la pensée de Ryan fut fortement influencée par de nouveaux courants de théologie catholique et la psychologie sociale dominant l'horizon de nombreux intellectuels américains. Sous son inspiration, l'Action catholique abandonne progressivement sa préoccupation initiale avec la réforme sociale, dominée par la «question des travailleurs» et la notion de la jeunesse comme classe à part dans la société moderne, pour une inquiétude centrée sur la classe moyenne québécoise, une orientation plus psychologique, centrée sur l'existence des mentalités religieuses et culturelles conflictuelles. Dès le milieu des années 1950, Ryan décerna dans ce conflit un vecteur dynamique de la sécularisation, mais il demeura plutôt optimiste que ces contemporains trouveraient, par le biais d'un catholicisme plus centré sur le spirituel, les ressources culturelles pour surmonter ce qu'il identifiait comme la «crise de l'adolescence» du Canada français.

1. Je voudrais particulièrement remercier Nancy Christie et Jean-Philippe Warren pour leurs commentaires et suggestions sur une première version du présent texte, ainsi que les évaluateurs anonymes de la revue. Je suis aussi reconnaissant au CRSH pour son appui financier. Bibliothèque et Archives nationales du Québec Montréal (BAnQM), Fonds Claude-Ryan (FCR), (P558, S12, SS1) Secrétaire national d'Action Catholique, 1995-12-001/361, Claude Ryan (CR), "Catholic Action in French Canada ", discours prononcé devant le groupe St. Benedict Labre, 16 octobre 1956.

2. BAnQM, FCR, (P558, S10, SS11), 2002-12-003/1, Carnet 1959, 3.2.59, John Henry Newman, Parochial and Plain Sermons, 208.16. 
ABSTRACT - This article explores the intellectual role played by Claude Ryan in a key cultural shift that occurred in Quebec's Catholic Action between 1945 and 1958. Influenced by new currents of Catholic theology and American social psychology, Ryan sought to move the organization from a preoccupation with social reform, dominated by the "labour question" in which youth was defined as a separate class in modern society, to a more psychological concern with clashing religious and cultural mentalities, centred on Quebec's rising middle class. In so doing, Ryan discerned a powerful vector of secularization, but remained hopeful that his contemporaries would find, in a more spiritually-conscious Catholicism, the cultural resources by which to navigate what he identified as the "adolescent crisis" of French Canada.

L a décennie qui suit la Deuxième Guerre mondiale a soulevé des difficultés importantes pour l'institution catholique québécoise. En dépit de taux élevés d'adhésion et de pratique religieuses (qui demeuraient parmi les plus élevés en Occident), l’Église était alors déchirée par de sérieuses tensions internes. Ces tiraillements avaient été générés par les stratégies et approches mises en place dans le but d'encourager un meilleur dialogue entre le catholicisme et la société canadienne-française.

Une bonne partie du problème découlait des querelles autour du rôle et de la place de l'Action catholique. Celle-ci regroupait une constellation de mouvements jeunesse spécialisés, lesquels avaient été établis avec l'approbation de la hiérarchie catholique au milieu des années 1930 dans le but explicite de rechristianiser les milieux sociaux et culturels fréquentés par la jeunesse urbaine et rurale. Fortement influencés par l'éthique "personnaliste", les mouvements jeunesse avaient eu tendance à s'émanciper du contrôle du clergé et à formuler une formidable critique des formes historiques qu'avait prises la relation du catholicisme à la société québécoise. Définissant le conflit en termes générationnels, ces mouvements remettaient en question en particulier la piété ritualiste de masse, l'omniprésence du clergé et des congrégations religieuses dans les domaines de l'éducation et de l'assistance sociale ainsi que l'adéquation irréductible entre catholicisme et identité canadienne-française ${ }^{3}$.

3. Pour une lecture de l'histoire du catholicisme québécois dans l'après-guerre en termes de conflits des interprétations, voir Michael Gauvreau, "Without making a noise” : the Dumont Commission and the Drama of Quebec's Dechristianization, 1968-1971 », dans Nancy Christie et Michael Gauvreau, dir., The Sixties and Beyond: Dechristianization in North America and Western Europe, 1945-2000 (Toronto, University of Toronto Press, 2013), 186-216. L'influence de l'éthique personnaliste sur le catholicisme québécois du $\mathrm{XX}^{\mathrm{e}}$ siècle a été étudiée par É.-Martin et Jean-Philippe Warren, Sortir de la grande noirceur: l'horizon personnaliste de la Révolution tranquille (Sillery, Septentrion, 2002); É.-Martin Meunier, Le pari personnaliste. Modernité et catholicisme au XX' siècle (Montréal, Fides, 2007). Pour une analyse de l'impact du personnalisme sur l'Action catholique, voir Michael Gauvreau, Les origines catholiques de la Révolution tranquille, 1931-1970 (Montréal, Fides, 2008, traduit par Richard Dubois; édition anglaise 2005). 


\section{POUR UNE POLITIQUE SPIRITUELLE}

À la fin de la guerre, la hiérarchie catholique a voulu réguler et discipliner davantage l'Action catholique en créant un bureau de coordination central, l'Action catholique canadienne (ACC) ${ }^{4}$. Ce nouvel organisme avait entre autres pour tâche de redéfinir la posture en termes de conflit des générations qui avait marqué les mouvements jeunesse spécialisés depuis leurs débuts. Il s'agissait de rompre avec l'idée selon laquelle la jeunesse non seulement constituait une "classe» à part, mais aussi affichait des intérêts forcément divergents par rapport à ceux de la génération précédente.

Appelés à choisir un secrétaire national qui allait donner corps et direction à l'ACC, les évêques surprirent bien des gens en arrêtant leur choix sur Claude Ryan. Âgé de 20 ans, ce dernier n'avait aucune expérience de l'Action catholique. Né à Montréal en 1925, il avait reçu, de 1937 à 1944, une éducation classique à l'externat Sainte-Croix. Sa formation intellectuelle initiale avait été très marquée par une veine «libérale» du nationalisme canadien-français (dont le journaliste Olivar Asselin était un des plus fameux représentants), par un "personnalisme» littéraire (puisé dans les ouvrages des écrivains français du début du $\mathrm{XX}^{\mathrm{e}}$ siècle, tels Charles Péguy et Joris-Karl Huysmans) et par la philosophie politique de Jacques Maritain. Le jeune Claude Ryan, suivant l'exemple d'un nombre important de ses contemporains, avait dévoré Humanisme intégral, paru en 1936. Il y avait trouvé un appel en faveur d'un militantisme spirituel tourné vers la construction d'un nouvel ordre chrétien qui se tenait à égale distance d'un capitalisme débridé et des systèmes totalitaires 5 .

Influencé par Maritain, le jeune Ryan avait rejeté la vocation sacerdotale à la fin de ses études à l'Externat Sainte-Croix. Voulant épouser la carrière d'un expert laïque, il avait préféré s'initier aux nouvelles disciplines des sciences sociales à l'École de service social de l'Université de Montréal. En 1945, après seulement un an, il quitta le programme sans même avoir obtenu son diplôme. Il fut immédiatement recruté par la hiérarchie catholique qui cherchait quelqu'un pour s'occuper du bureau national de l'Action catholique.

4. Louise Bienvenue, Quand la jeunesse entre en scène. L'Action catholique avant la Révolution tranquille (Montréal, Boréal, 2003).

5. On trouvera des renseignements sur la formation intellectuelle de Ryan dans Michael Gauvreau, "Catholicisme, nationalisme, et fédéralisme dans la pensée de Claude Ryan: la contribution de l'Action catholique, 1945-1964", Revue d'histoire de l'Amérique française, 62, 3-4 (hiver-printemps 2009), 429-472; Michael Gauvreau, "Les "nouveaux" réseaux catholiques de Claude Ryan: le changement de cap de l'Action catholique canadienne (1950-1962)", Études d'histoire religieuse, 79, 1 (2013): 45-58. 
En désignant Ryan, les évêques évinçaient des porte-parole connus et reconnus de la jeunesse québécoise, dont Gérard Pelletier, Guy Rocher et Pierre Juneau, qui avaient tous passé par les rangs de la Jeunesse étudiante catholique (JEC). Sa nomination semblait néanmoins justifiée. En premier lieu, comme il n'entretenait pas de liens avec la JEC, Ryan ne souscrivait pas à l'idée, propagée depuis les débuts de l'Action catholique spécialisée, d’un conflit générationnel. Les mouvements spécialisés du Canada (qui ciblaient les étudiants, ainsi que les jeunes des milieux ruraux et ouvriers) restaient marqués par la Grande Crise. On soutenait encore que la meilleure façon de combattre le communisme, ici ou à l'étranger, était d'intéresser de près les jeunes catholiques canadiens-français aux enjeux sociaux ${ }^{6}$. Ensuite, on affirmait que la jeunesse constituait, dans les sociétés modernes, une sous-classe et que celle-ci avait un besoin pressant d'institutions et de structures propres afin de pallier ses désavantages sociaux et culturels.

Bien différente, l'approche de Ryan paraissait être sagement ancrée dans l'idéologie libérale nord-américaine. Par exemple, quoique tournée vers l'aide à la jeunesse urbaine défavorisée, sa conception du travail social ne faisait pas des jeunes une «classe » dont les intérêts auraient été divergents par rapport à la société adulte. Ryan regardait davantage les jeunes comme des "citoyens en devenir", c'est-à-dire comme des individus qui avaient besoin d'une bonne éducation, d'une solide préparation au mariage ainsi que d'une rigoureuse formation professionnelle s'ils souhaitaient un jour fonder une famille.

En deuxième lieu, alors que des hommes comme Pelletier avaient, pendant la Deuxième Guerre mondiale, ouvertement critiqué l'effort de guerre canadien et, implicitement, la hiérarchie catholique qui avait fermement appuyé le gouvernement, Ryan s'était abstenu de telles récriminations. Bien que réformé pour des raisons médicales, Ryan pouvait se targuer d'appartenir à une famille à la feuille de route impeccable: ses deux frères s'étant portés volontaires. Finalement, il était parfaitement bilingue, ce qui constituait un atout important. À cette époque, les autorités catholiques du Québec souhaitaient travailler aux initiatives de reconstruction de l'aprèsguerre de concert avec les organisations anglo-canadiennes. La Commission canadienne de la jeunesse exprimait cette volonté de coordination et de concorde ${ }^{7}$.

6. L. Bienvenue, op. cit., 200.

7. Pour une analyse des motifs ayant conduit au choix de Ryan en 1945, voir Michael Gauvreau, $A$ Parallel Government of the Spirit: Claude Ryan and the Fate of Canadian Liberalism, 1925-1971 (Toronto, 
À l'ACC, la tâche de Ryan consistait grosso modo à coordonner un foisonnement de mouvements et d'agences. On retrouvait de tout dans l'ACC: des services de presse, des journaux, des magazines, des camps d'été, des centres pour les jeunes, des agences de placement, un mouvement de préparation au mariage florissant, des coopératives d'habitation. Les activités de l'ACC participaient au rêve d'instaurer une «société catholique parallèle». Créées dans les années 1930, ces structures représentaient la réponse du laïcat à l'appel d'une Église qui cherchait à incarner sa doctrine sociale dans la société moderne. Elles s'étaient ensuite adaptées aux initiatives populaires venues des gens de la classe moyenne inférieure et de la classe ouvrière qui voulaient se donner divers services en l'absence d'un État-providence. On peut aussi avancer que, axées sur les priorités sociales et culturelles des jeunes hommes et des jeunes femmes, ces actions constituaient une sorte d'expérience continue de démocratie sociale et civique ${ }^{8}$.

Quoi qu'il en soit, l'ambition de Ryan allait bien au-delà de ce qu'imaginaient ses supérieurs ecclésiastiques. Ryan nourrissait l'espoir de faire de l'ACC un centre où pourraient se réunir les intellectuels canadiensfrançais de tous âges. Il entrait dans ce souhait un certain sentiment d'infériorité par rapport à d'autres jeunes de son âge (pensons à Guy Rocher, Fernand Dumont, Gérard Pelletier, Pierre Elliott Trudeau et Maurice Sauvé) qui avaient acquis ou étaient en voie d'acquérir des diplômes dans des établissements prestigieux à Londres, Paris, Chicago et Harvard. Ryan, lui, ne réussira jamais à compléter sa formation universitaire. Par conséquent, il se voyait davantage comme l'héritier du mouvement des Jeune-Canada lancé par André Laurendeau et Gérard Filion dans les années 1930. Il espérait forger un équilibre dynamique entre le nationalisme, le catholicisme et les tendances réformistes, équilibre qui semblait seul en mesure d'orienter l'engagement des jeunes hommes conscientisés ${ }^{9}$. Quoique son poste exigeait de lui qu'il demeure rigoureu-

University of Toronto Press, sous presse), chapitre 2. Pour en savoir davantage sur la Commission canadienne de la jeunesse, voir Michael Gauvreau, «The Protracted Birth of the Canadian "Teenager”: Work, Citizenship, and the Canadian Youth Commission, 1943-1955 ", dans Nancy Christie et Michael Gauvreau, dir., Cultures of Citizenship in Postwar Canada, 1940-1955 (Montréal \& Kingston, McGill-Queen's University Press, 2003), 201-238.

8. Là-dessus, voir M. Gauvreau, Les origines catholiques..., op. cit.; Lucie Piché, Femmes et changement social au Québec: l'apport de la Jeunesse ouvrière catholique féminine, 1931-1966 (Québec, Les Presses de l’Université Laval, 2003).

9. Le sentiment de ne pas être à sa place et les initiatives subséquentes de Ryan pour susciter l'engagement de ses confrères intellectuels ont été analysés par M. Gauvreau, "Les "nouveaux” réseaux catholiques ...» loc. cit., 49-50. 
sement "apolitique», Ryan cherchait à formuler les termes d'un engagement collectif qui puisse retisser les liens entre le catholicisme et la société canadienne-française. Cette stratégie culturelle lui semblait en mesure d'alléger les tensions qui existaient entre les intellectuels de son milieu, tensions qui avaient commencé à émerger à partir du milieu des années $1940^{10}$.

Les intellectuels canadiens-français étaient de plus en plus combatifs dans les années 1950. Cependant, ceux que côtoyait Ryan étaient toujours unis par la volonté de conserver au catholicisme sa place centrale dans l'identité du Canada français. Ces intellectuels catholiques entraient régulièrement en conflit avec l'Église institutionnelle, critiquant tout particulièrement quelques-unes des activités du clergé dans la sphère temporelle $^{11}$. Comme eux, Ryan était persuadé que l'Action catholique ne pourrait devenir un point de ralliement qu'à la condition de mettre de l'avant les valeurs du catholicisme. Il trouvait dans cette conviction la clé de sa propre identité comme intellectuel et comme médiateur culturel. En effet, contrairement au Canada anglais, le Québec offrait au catholicisme un véritable espace de dialogue et une voie possible pour canaliser l'engagement des intellectuels.

Cette place laissée au catholicisme se rapprochait de ce que l'on observait au même moment aux États-Unis. À la fin des années 1940 et au début des années 1950, les catholiques américains formulaient un type de critique sociale et de commentaire culturel qui s'appuyait sur la possibilité d'une "politique spirituelle». Ils pensaient pouvoir faire de la religion une dimension intégrale du système culturel et l'élever à la hauteur d'une "foi nationale ${ }^{12}$ », spirituellement inclusive. Ils croyaient que ce consensus pourrait contenir les tensions croissantes générées, dans l'après-guerre, par une modernisation accélérée. Une semblable promotion du catholicisme comme "politique spirituelle» sous-tendait les activités de Ryan dans les années 1950. L'idée que cette "politique spirituelle» pourrait unifier et régénérer la société canadienne-française allait guider ses choix

10. Pour la catégorisation de la communauté intellectuelle en "nationaliste traditionaliste», «néonationaliste » et "anti-nationaliste», voir Pascale Ryan, Penser la nation. La Ligue d'action nationale, 1917-1960 (Montréal, Leméac, 2006), 224-230, 241; Jean-Philippe Warren, L'engagement sociologique. La tradition sociologique du Québec francophone (1886-1955) (Montréal, Boréal, 2003), 245-253, 256-269 ; Xavier Gélinas, La droite intellectuelle québécoise et la Révolution tranquille (Québec, Les Presses de l'Université Laval, 2007); Michael Behiels, Prelude to Quebec's Quiet Revolution: Liberalism versus Neo-Nationalism (Montréal \& Kingston, McGill-Queen's University Press, 1985).

11. Pour une analyse plus détaillée, voir M. Gauvreau, Les origines catholiques..., op. cit., 39-82.

12. Mark Silk, Spiritual Politics: Religion and America since World War II (New York, Simon and Shuster, 1988), 19. 
et définir ses alliances politiques jusqu'à la fin de la Révolution tranquille.

\section{Catholicisme et problème de la civilisation}

Ryan avait fait la «découverte» déterminante de la réalité de l’Église universelle pendant son séjour d'études à la Grégorienne, l'université pontificale de Rome, en 1951-1952. L'étude intensive de l'histoire de l'Église et de l'ecclésiologie lui avait fait connaître la "nouvelle théologie» augustinienne d'Yves de Montcheuil, Henri de Lubac et Yves Congar. Ce courant intellectuel avait renforcé chez lui une posture pragmatique. Il y avait trouvé une réponse à la question de l'orientation de l'Action catholique dans le nouveau contexte créé par la grève d'Asbestos, c'est-à-dire à un moment où la hiérarchie ecclésiale regardait avec suspicion toute expression de sympathie pour la gauche progressiste.

L'approfondissement de la théologie augustinienne avait convaincu Ryan de la fausseté d'une philosophie purement rationnelle et sécularisée de l'histoire (comme celles proposées par le libéralisme et le marxisme dans l'entre-deux-guerres). Mais si, pour Ryan, le sens de la destinée humaine n'était accessible que pour ceux qui la scrutaient avec les yeux de la foi, il reste que les civilisations passées tout autant que les progrès de l'Amérique du Nord et de l'Europe de l'Ouest démontraient les affinités étroites qui existaient entre le christianisme et la civilisation occidentale. "Le catholicisme, rappelait Ryan à un groupe d'étudiants de l'Université de Montréal à l'automne 1952, reste le premier - peut-être aussi le seul et dernier - espoir de l'Europe ${ }^{13}$.»

La théologie de l'histoire de Ryan était consolidée par la lecture des grandes fresques historiques brossées par les écrivains britanniques Arnold Toynbee et Christopher Dawson. Les travaux de ces auteurs confirmaient l'intuition augustinienne: l'histoire était réellement "the masterful and progressive execution [...] of a divine plan [...] which transcends our human powers of vision and understanding in every dimension». Ils confirmaient aussi que les hommes modernes vivaient un conflit spirituel d'une rare profondeur. Le problème était crucial pour les élites intellectuelles occidentales, spécialement pour les classes moyennes des démo-

13. BAnQM, FCR, Secrétaire national d'Action Catholique (P558, S12, SS1), 1995-12-001/359, Claude Ryan, «Le catholicisme en Europe», notes pour une causerie à l’Équipe UdeM de l'A.C., 25 octobre 1952; Ibid., 1995-12-001/359, Claude Ryan, "Théologie de l’histoire», notes sur un cours enseigné par Joseph Folliet, Université de Montréal, 3 octobre 1952. Pour en connaître plus sur le voyage de Ryan à Rome, lire M. Gauvreau, «Les "nouveaux” réseaux catholiques de Claude Ryan... » loc. cit., 51-4, M. Gauvreau, A Parallel Government of the Spirit..., op. cit., chapitre 3. 
craties industrielles, cette "creative minority " dont les initiatives «created the modern world». L'incapacité à résoudre le conflit spirituel auquel elle était confrontée causerait inévitablement la désintégration de la civilisation occidentale ${ }^{14}$. Pour Dawson, le leadership culturel était de plus en plus compromis par ce qu'il appelait une «schizophrénie sociale». Le divorce allait croissant entre une vision du monde scientifique de plus en plus amorale et un système religieux et moral de croyances qui n'avait que peu d'emprise sur l'existence humaine. Toutefois, Dawson ne plaidait pas pour l'établissement d'une nouvelle religion. Il invoquait plutôt la nécessité d'accomplir une réintégration spirituelle, ce qui, chez lui, voulait dire restaurer une "relation vitale» entre la religion et la culture moderne ${ }^{15}$.

Ryan a été marqué par sa lecture de Toynbee et Dawson ainsi que par sa conversion à une théologie de l'histoire conflictuelle. Il en a tiré une nouvelle mission pour l'Action catholique, mission qui éloignait le mouvement de la "question sociale». Il ne s'agissait plus, comme cela avait été le cas durant la modernité industrielle, de forger une nouvelle chrétienté à partir d'une réforme des structures de la société capitaliste. Il fallait désormais plaider pour une restauration des critères esthétiques et culturels. On se souciait moins d'un assouplissement des relations entre les classes sociales et davantage de la qualité de la vie intérieure des membres des classes moyennes. Sollicité pour accomplir cette tâche difficile, Ryan croyait qu'il pouvait accomplir immédiatement le mandat de la hiérarchie épiscopale. Dépolitisant l'Action catholique, il lui donnait une mission culturelle et spirituelle davantage appropriée à une société nord-américaine qui, soulevée par une vague sans précédent de prospérité, était de plus en plus attentive aux dimensions culturelles de l'existence humaine.

À partir de 1953, le discours de Ryan trahit une nouvelle urgence. Ce dernier tenait à distancier l'Action catholique de la gauche progressiste, laquelle souhaitait comme toujours transformer les relations entre les classes et les hiérarchies qui structuraient les lieux de travail. Dans son premier article publié dans Le Devoir, Ryan identifiait les changements ayant affecté le milieu canadien-français depuis 1945. Il déclarait que, désormais, l'enjeu qui devait préoccuper les intellectuels était moins l'équilibre changeant des forces politiques à l'intérieur ou à l'extérieur de la province de Québec, ou l'urbanisation du Canada français, mais le «changement enfin

14. Arnold J. Toynbee, Civilization on Trial (New York, Oxford University Press, 1948), 14-15, 21; Christopher Dawson, Religion and Culture. Gifford Lectures delivered at the University of Edinburgh in the year 1947 (Londres, Sheed and Ward, 1948), 216-17.

15. C. Dawson, Religion and Culture, op. cit., 216-218. 
dans l'équilibre de notre vie religieuse ", et tout particulièrement la prise en compte des réalités religieuses par le laïcat ${ }^{16}$. Prenant la parole devant le conseil national de la Jeunesse ouvrière catholique en 1953, Ryan cherchait à convaincre les militants catholiques issus de la classe ouvrière de se tenir loin du radicalisme politique. Il agitait le mot d'ordre de l'obéissance à la fois à lui-même, comme secrétaire national, et aux évêques: «l'autorité est d'abord un service, l'obéissance une forme supérieure d'épanouissement et le détachement la forme la plus active et la plus efficace de présence ${ }^{17}$ ».

Bien que les discours que Ryan adressait aux militants catholiques fussent remplis d'exhortations à cultiver une "intériorisation plus intense» du message chrétien et une "expérience communautaire plus authentique» de la foi, il n'était pas question pour lui de revenir à une piété traditionnelle plus paisible ${ }^{18}$. Aux critiques (venus de l'intérieur et de l'extérieur de l'Action catholique) qui se plaignaient que l'Église entrait dans une phase réactionnaire et conservatrice, Ryan répliquait que l'Action catholique traversait une période d'ajustement aussi pénible que nécessaire. Le laïcat était désormais invité à cultiver une "Autonomie pour la responsabilité $^{19}$ ", ce qui représentait un correctif par rapport à l'ancienne approche de l'Action catholique qui avait cherché à infuser de manière trop mécanique les valeurs catholiques.

Reprenant l'analogie (courante dans les écrits du catholicisme social) du corps humain, on avait traditionnellement défini le laïcat comme une sorte de «membre» externe qui avait pour tâche d'exécuter les ordres donnés par le "cerveau » clérical. Ryan décriait cette approche. Il y voyait une forme de «cléricalisme grossier» qui était, heureusement, en voie de disparaittre ${ }^{20}$. Ryan proposait un grand virage à l'Action catholique. Il incitait ses membres à ne plus s'investir autant dans le redressement de la classe ouvrière et à épouser une mission davantage culturelle. Il s'agissait d'amener à une existence spirituelle plus vigoureuse les membres des classes moyennes canadiennes-françaises, et en particulier ceux apparte-

16. Claude Ryan, "Après sept années d'expériences...: Les conférences du Devoir», Le Devoir, $1^{\mathrm{er}}$ octobre 1953.

17. BAnQM, FCR, (P558, S12 SS1) Secrétaire national d'Action Catholique, 1995-12-001/359, Claude Ryan, "L’unité de pensée et d'action en J.O.C., résumé d'exposé donné au conseil national de la J.O.C.», novembre 1953.

18. BAnQM, FCR, (P558, S12, SS1) Secrétaire national d'Action Catholique, 1995-12-001/359, Claude Ryan, "Talk to Ordre du Bon Temps» (O.B.T.), 5 septembre 1953.

19. BAnQM, FCR., (P558, S10, SS11), 2002-12-003/1, Carnet 3, 1953. Souligné dans l'original.

20. BAnQM, FCR., (P558, S12, SS1), Secrétaire national d'Action Catholique, 1995-12-001/360, Claude Ryan, "Causerie à réunion des aumôniers diocésains de J.O.C. et L.O.C., Sainte-Agathe », 6 octobre 1954. 
nant aux nouvelles professions: enseignants, infirmiers, journalistes, spécialistes des médias et intervenants sociaux.

Ryan était persuadé que les stratégies du passé comportaient peu d'attrait pour ceux qui détenaient une formation secondaire ou universitaire, ou encore un diplôme professionnel avancé. Il ne croyait pas au succès des méthodes d'un père Joseph-Papin Archambault, le fondateur jésuite de l'École sociale populaire, qui cherchait encore, dans les années 1950, à faire retrouver au catholicisme son influence en organisant des croisades morales. Écrivant au père Archambault en 1953, Ryan refusait catégoriquement de mettre l'Action catholique au service d'une campagne contre le père Noël. Il soutenait que l'organisation souhaitait éviter, d'une part, de participer à des campagnes massives de moralité et, d'autre part, de créer une panoplie de services spécialisés qui entraîneraient de lourdes dépenses ${ }^{21}$.

La légitimité des nouveaux professionnels canadiens-français reposait dorénavant sur la formation et l'expertise. S'affirmant comme le groupe clé de l'Action catholique, ces professionnels ne seraient pas mobilisés par le martèlement de directives autoritaires. "Le monde de la science et de la technique, disait Ryan, doit être transformé du dedans. Impossible de Xiser [christianiser] ces domaines sans la compétence et l'expérience ${ }^{22}$.» Il n'était plus question non plus, pour l'Action catholique, de chercher à se substituer à l'État et à investir la sphère publique, comme cela avait été le cas naguère. Ryan déclarait: «Elle [l'Action catholique] doit revenir sur son terrain propre qui est celui de la sanctification de la transmission du divin.» Le mouvement devait poursuivre son action sur le terrain social, mais cette action devait maintenant être "indirecte et non calculée; elle jaillit de l'action temporelle des laïcs élevés par l'Action catholique au sens

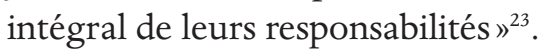

Mais comment attirer les nouveaux groupes professionnels dans l'orbite de l'Action catholique? Quand on consulte les textes que Ryan a écrits à son retour de Rome, un adjectif revient sans cesse: "réaliste». Dans le climat de l'après-guerre, ce mot avait une résonance particulière chez les jeunes professionnels. Les calepins de notes de Ryan, de même que ses notes de réunions et de conversations avec des membres du clergé et des

21. Service des Archives de l'Université de Montréal (SAUM), Fonds Action Catholique Canadienne (FACC), P16/I.95, «Institut Social Populaire», Claude Ryan au père Joseph-Papin Archambault, 23 avril 1953.

22. BAnQM, FCR, (P558, S10, SS11), 2002-12-003/1, Carnet 3, 1953.

23. Claude Ryan, "Les mouvements d'apostolat laïc dans l'Église», dans Centre catholique des intellectuels canadiens, Le rôle des laïcs dans l'Église. Carrefour 1951 (Montréal, Fides, 1951), 77. 
leaders laïques, sont remplis de mises en garde contre la tentation de mesurer la vitalité spirituelle à l'aune d'un recrutement de masse ${ }^{24}$. Les élans collectifs, arguait Ryan, sont trop basés sur l'émotion et l'enthousiasme spontané, et pas assez sur la réflexion et l'analyse. Pour responsabiliser les mouvements d'Action catholique, il paraissait donc important de laisser les laïques débattre librement des questions théologiques et des sujets religieux. Cette liberté leur permettrait, en retour, d'élaborer une synthèse personnelle qui orienterait leur action et leur spiritualité25.

Au dire de Ryan, deux obstacles compromettaient l'ouverture de l'Action catholique aux classes moyennes. En premier lieu, l'Action catholique n'avait pas été en mesure d'offrir aux hommes éduqués un climat intellectuel stimulant, ce qui avait eu pour conséquence d'appauvrir l'expérience militante. La trajectoire personnelle de Ryan était l'exception qui confirmait la règle. Il avait tiré avantage du fait que sa vie était caractérisée par "certaines ambiguïtés» que ses supérieurs épiscopaux n’avaient pas pris le temps de sérieusement considérer: il était encore célibataire, et des circonstances familiales lui avaient permis d'entretenir d'excellentes relations avec le clergée ${ }^{26}$. Dans le contexte du Canada français des années 1950, Ryan jugeait que l'on pouvait au mieux espérer faire évoluer l'Action catholique vers un "cléricalisme éclairé». Les dirigeants des bureaux nationaux et diocésains avaient encore besoin de s'appuyer sur l'autorité des membres compétents du clergé en attendant l'accession des laïques à la maturité spirituelle ${ }^{27}$.

Le deuxième obstacle venait des tensions qui s'étaient développées entre la structure de l'Action catholique et l'existence des hommes modernes. Les gens travailleraient dans des organisations de plus en plus anonymes. Dès lors, la question se posait de savoir comment des mouvements encadrés par une Église autoritaire pouvaient transcender la dépersonnalisation et l'aliénation auxquelles les individus étaient sujets dans leurs vies professionnelles quotidiennes. Ryan aimait retourner aux écrits d'un de ses mentors de l'entre-deux-guerres, l'écrivain personnaliste Charles Péguy. Il reprenait

24. BAnQM, FCR, (P558, S10, SS11), 2002-12-003/1, Carnet 3, 1953; Ibid., Carnet 5, 1956, M ${ }^{\mathrm{gr}}$ Morin, 16.5.56; BAnQM, FCR, (P558, S12, SS1), Secrétaire national d'Action Catholique,1995-12-001/359, Claude Ryan, «Études ecclésiologiques - Promotion du laïcat, »ca. 1952-53, Cardinal Newman, «An Instructed and Articulate Laity", extrait de La Voix du Vicariat de Grouard, janvier 1953.

25. BAnQM, FCR, (P558, S12, SS1), Secrétaire national d'Action Catholique, 1995-12-001/359, Claude Ryan, "Attitudes du laïc dans l'A.C.», notes pour une Causerie à Aumôniers locaux de L.O.C., Montréal, 30.8.53.

26. BAnQM, FCR, (P558, S12, SS1), Secrétaire national d'Action Catholique, 1995-12-001/359, Claude Ryan, «Rapports entre clergé et laïcs au Canada», exposé à la Maison Léon XIII Team, 15 février 1954.

27. Ibid. 
son fameux dicton selon lequel les mouvements avaient tendance à commencer par une puissante impulsion spirituelle, avant de dégénérer ensuite en slogans politiques et en compromis pragmatiques: «tout commence en mystique et finit en politique», disait Péguy. Ryan reprenait cette observation afin de mieux dénoncer les rigidités de la bureaucratie et l'horizon borné de la vie intellectuelle moderne: "Ce qui commence en mystique finit en administration», affirmait-il avec des accents néo-weberiens.

Dans l'ensemble de ce qu'était devenue l'Action catholique, Ryan discernait les symptômes d'un étiolement institutionnel. Les directeurs du mouvement n'étaient pas représentatifs de leur milieu social; les leaders étaient coupés des membres qu'ils devaient prétendument servir; les aumôniers et les leaders étaient imposés par le haut et tendaient à rester en poste trop longtemps; il existait des tendances alarmantes au corporatisme, ce qui se manifestait par la volonté de certains mouvements d'être cooptés par l'État; les questions relatives à la spiritualité étaient largement absentes $^{28}$. Mais, contrairement au sociologue allemand Max Weber, pour qui il n'y avait aucune issue à la loi de fer de la bureaucratie moderne, Péguy et Ryan pensaient qu'il existait un remède à l' "embourgeoisement» de l'Église. La solution se trouvait dans la personnalité charismatique de l'intellectuel. La réponse authentique à l'appel de la foi pouvait insuffler à nouveau le dynamisme de la «mystique» dans des structures vermoulues et ainsi renverser les pressions organisationnelles de la vie moderne qui, autrement, conduisaient à l'habitude et la routine ${ }^{29}$.

\section{La sécularisation comme esthétique: Ryan et le drame spirituel du Canada français}

Dans le projet culturel de Ryan, l'intellectuel recevait la tâche de revitaliser la religion et de lui redonner son authenticité. La ligne de faille de la société canadienne-française ne passait plus par des divisions de classes, comme l'avaient supposé les membres de l'Action catholique depuis la Grande Dépression. Aux yeux de Ryan, la fracture entre les deux principales tendances spirituelles du Canada français paraissait davantage suivre des oppositions esthétiques et psychologiques.

Sur ce point, Ryan était parfaitement en phase avec un des discours dominants de la société américaine de l'après-guerre. Ce discours était énoncé autant par des intellectuels conservateurs que des intellectuels libéraux et radicaux. À la critique des inégalités créées par le capitalisme

28. BAnQM, FCR, (P558, S10, SS11), 2002-12-003/1, Carnets 1953.

29. Sur l'importance de Péguy, voir M. Meunier, Le pari personnaliste..., op. cit., 101-103, 121. 
était désormais substituée celle de l'homogénéisation de la culture de masse. On affirmait que, en Amérique du Nord, les clivages fondamentaux suivaient désormais des lignes culturelles plutôt que sociales, ce qui avait notamment pour conséquence d'éloigner les intellectuels des polémiques politiques. Le statut des intellectuels était rehaussé par la conviction que, dans le monde moderne, seule leur lucidité permettrait d'éviter la déshumanisation créée par le conformisme, et donc, potentiellement, l'avènement de régimes autoritaires, voire totalitaires. Le monde avait besoin d'hommes animés par une esthétique moderniste, culturellement raffinée $^{30}$. Dans l'esprit de Ryan, les catégories de culture «populaire» et de culture «élitiste» renvoyaient à des sensibilités religieuses concurrentes, sensibilités qui traversaient le catholicisme contemporain.

Un premier aspect qui distinguait, dans les années 1950, les polémiques au Québec et celles qui se déroulaient aux États-Unis, c'était que, dans la province, la religion était le terrain même sur lequel se manifestait le clash entre tradition et modernité. Un deuxième aspect distinguait la pensée de Ryan par rapport à ses confrères américains. À ses yeux, une «culture de masse" faisait peser tout le poids de la tradition et de l'immobilisme à l'intérieur même du catholicisme. Cette culture de masse n'était pas spécifique à une classe sociale. Elle consistait en un état socio-psychologique qui tendait à accoucher d'un tempérament religieux routinier, justifiait un anti-intellectualisme infantile et favorisait une adhésion prémoderne aux attributs matérialistes de la religion. Pour empêcher ces dérives, Ryan liait la culture catholique d'une élite moderniste à la quête personnaliste de la "nouvelle théologie». Il cherchait à montrer que les racines historiques et bibliques de la foi chrétienne plongeaient dans un terreau résolument progressiste. Il voulait promouvoir les valeurs d'un engagement spirituel adulte, alimenté aux sources d'une foi authentique et basée sur les canons de la théologie moderne et une valorisation de l'expertise.

Les nouvelles classes moyennes se débattaient dans un «drame spirituel». Ce drame opposait les hommes et les femmes, les jeunes et les vieux ainsi que l'élite et la masse de la société canadienne-française. Modelées dans les forges de la modernité industrielle, les solutions anciennes ne

30. Howard Brick, Daniel Bell and the Decline of Intellectual Radicalism: Social Theory and Political Reconciliation in the 1940s (Madison, University of Wisconsin Press, 1986), 39, 52 ; dans Christian Critics: Religion and the Impasse in Modern American Social Thought (Ithaca et London, Cornell University Press, 2000), 104, Eugene McCarraher souligne une tendance à esthétiser les conflits sociaux afin de les présenter comme affaires de goût plutôt que comme des enjeux de pouvoir et de moralité. Voir aussi Gary Sumner, Dwight Macdonald and the politics Circle: The Challenge of Cosmopolitan Democracy (Ithaca et London, Cornell University Press, 1996), 6-40. 
résonnaient plus dans le monde contemporain. Il était hors de question de reprendre telles quelles les formules aussi simplistes qu'obsolètes offertes naguère par les idéologies conservatrices ou progressistes. Aux membres de l'Action catholique, et en particulier aux penseurs catholiques de la lignée de Ryan, revenait la tâche ô combien importante de trouver un principe qui puisse restaurer l'unité brisée du milieu intellectuel canadien-français. Ils pourraient ainsi guider leur société vers la maturité spirituelle, ce qui n'adviendrait que s'ils réussissaient «à s'incorporer le drame qui se joue actuellement dans la conscience du milieu travaillé par son évolution historique ${ }^{31} »$.

Au milieu des années 1950, un leitmotiv revient dans les écrits de Ryan lorsqu'il est question de définir la relation du catholicisme à la société canadienne-française: devant des auditoires qui n'étaient pas seulement composés de membres de l'Action catholique, Ryan rappelait que sa société traversait une "crise» aux ramifications à la fois spirituelles et culturelles. Nous devons toutefois être prudents avant de sauter à la conclusion qu'il était pessimiste quant à la possibilité de conserver au catholicisme une place centrale dans la constitution de l'identité canadienne-française. Ryan rappelait, en 1953, avoir discuté avec le pasteur de l'Église unie, Ted Nichols, un ami canadien-anglais, des problèmes auxquels faisait face l’Église du Québec. Il confiait pouvoir parler à Nichols "franchement comme à un frère». Or, Nichols lui avait dit de ne pas se soucier outre mesure des conflits internes de l'Église car, pour lui, de tels conflits devaient être vus comme des signes de vitalité. Ils offraient un heureux contraste lorsqu'ils étaient comparés à la vie intellectuelle somnolente des Églises protestantes canadiennes ${ }^{32}$. Dans l'esprit de Ryan, le terme «crise» revêtait donc (au moins jusqu’au début des années 1960) une connotation largement positive. Il désignait de manière globale, c'està-dire pour la société tout entière, la lutte que des adolescents étaient en train de livrer pour accéder à la maturité émotionnelle et intellectuelle.

À ses lectures diverses qui portaient sur la théologie et l'histoire catholiques, Ryan ajoutait le prestige culturel de la psychologie américaine. Dans les années 1950, cette discipline recevait par monsieur et madame tout-le-monde une interprétation presque exclusivement psychanalytique. Cette psychologie, notons-le, était fondée sur un système de dichotomies qui opposaient le plus couramment les traits masculins aux traits féminins

31. SAUM, FACC, P16/B4, 1,1, «Évolution de l'ACC entre 1945 et 1952», janvier 1950-février 1953, "Bilan du travail accompli par le Premier Comité National de l'A.C.C. », février 1953.

32. BAnQM, FCR, (P558, S10, SS11), 2002-12-001/1, Carnets 1953. 
(les premiers étant jugés «rationnels» et normatifs), la tradition à la modernité ainsi que l'individualisme au conformisme ${ }^{33}$. Ryan croyait que, au Canada français, ces oppositions structuraient le devenir du catholicisme lui-même. Il aurait été étonnant qu'il en soit autrement, au moins en surface, considérant la profonde affinité qui existait entre les normes institutionnelles catholiques et la pratique religieuse de la plupart des Canadiens. Cependant, pour un Augustinien comme Ryan, qui aimait les paradoxes et les dualismes, l'Église comprenait irrévocablement deux systèmes culturels, l'un orienté vers le passé et la tradition, et l'autre tourné vers l'avenir et la modernité.

$\mathrm{Vu}$ ainsi, le discours de Ryan ne détonnait pas par rapport à celui des collaborateurs anticléricaux de Cité libre, des étoiles montantes de la tradition sociologique de l'Université Laval ou des néonationalistes regroupés autour du Devoir. Ce qui rassemblait tous ces groupes dans une même communauté de pensée, c'était la conviction que, si le catholicisme représentait un des socles de l'identité culturelle du Canada français, il devait être conjugué avec les valeurs de la modernité (lire: ouverture, compétence scientifique et universalité) et ne pas se résorber en un système clos de valeurs ethniques. Pour que la société canadienne-française soit capable de traverser avec succès cette crise, il fallait formuler une nouvelle synthèse entre catholicisme et culture qui soit en mesure de susciter l'engagement de citoyens les plus modernes, c'est-à-dire ceux vivant dans des sociétés de masse. C'était, pour Ryan, la seule façon de faire parvenir le Canada français à la pleine maturité sociale et culturelle, par quoi il voulait dire l'acceptation de standards culturels universaux en remplacement des formes régionales particularistes et "arriérées ».

Ryan a tenté de diagnostiquer cette "crise». Au début de 1955, il avait été invité à livrer un discours aux membres de la presse étudiante universitaire et collégiale dans le château fort de Maurice Duplessis, TroisRivières. Ce discours mérite une attention particulière parce que Ryan changera peu son angle d'approche dans le reste de la décennie. On y retrouve un diagnostic quant à la meilleure façon, pour le Canada français, d'atteindre la plénitude spirituelle et culturelle, de même que l'identification des principaux symptômes du mal qui ronge sa société, leur étiologie ou leur analyse générale. Il faut souligner que l'homme n'affiche pas ici une grande originalité. Il ne faisait que reprendre des thèmes familiers

33. James Gilbert, Men in the Middle: Searching for Masculinity in the 1950s (Chicago, University of Chicago Press, 2005), 62-80. 
aux lecteurs de la littérature personnaliste de France, auxquels il ajoutait le vocabulaire de la science américaine. L'importance de son propos vient du fait qu'il était prononcé par un homme qui était, volens nolens, le bras droit de la hiérarchie catholique du Québec et son principal conseiller laïque sur les questions sociales - une affirmation qui ne vaut pas pour les intellectuels de Cité libre, leurs diatribes anticléricales ayant terni leur réputation parmi le clergé et les milieux catholiques dévots.

À l'évidence, les opinions formulées par Ryan dans son discours avaient déjà obtenu une circulation considérable dans la communauté des intellectuels canadiens-français ${ }^{34}$. Même le titre de son exposé, «La Rencontre de deux mondes ", faisait explicitement référence au travail du célèbre sociologue américain Everett C. Hughes (lequel avait aidé à lancer le programme de recherche à la Faculté des sciences sociales de l'Université Laval). Inspiré par les travaux des intellectuels plus jeunes, comme JeanCharles Falardeau et Fernand Dumont, Ryan opposait les valeurs et pratiques de la société "folk» traditionnelle à celles de la "société urbaine». Il pensait que le développement de relations sociales propres à la civilisation industrielle avait directement conduit au "cultural lag» (qui désigne le retard culturel de la superstructure idéologique par rapport à l'infrastructure économique) dont souffrait le Canada français dans l'aprèsguerre. Cette opinion était exprimée de manière véhémente, au milieu des années 1950, par les membres de «l’École de Montréal» : les historiens appartenant à cette École affirmaient que le «retard» et l’infériorité économique persistante du Canada français par rapport à l'Amérique du Nord de langue anglaise étaient attribuables à l'influence excessive des valeurs et des institutions catholiques ${ }^{35}$.

Ryan avait choisi de commencer son discours par l'affirmation percutante que «le peuple canadien-français traverse une crise de conscience qui affecte tous les domaines, culturel, économique, national, religieux, politique. Cette crise est un fait réel. » Les intellectuels devaient se sentir interpelés par cette crise: non seulement le Canada français se trouvait divisé de l'intérieur mais, de surcroît, aucune des utopies traditionnelles avancées par le clergé, les partis politiques ou l'entreprise privée ne pouvait inspirer un projet social ou culturel en mesure de résorber cette frac-

34. M. Gauvreau, Les origines catholiques de la Révolution tranquille..., op. cit., 67-71.

35. J.-P. Warren, L'engagement sociologique..., op. cit., 285-290. Au sujet de la thèse du "retard» du Canada français qui a dominé les travaux des historiens de l’Université de Montréal, voir Jean Lamarre, Le devenir de la nation québécoise, selon Maurice Séguin, Guy Frégault, et Michel Brunet (1944-1969) (Sillery, Septentrion, 1993), 18-19. 
ture. «Il faut, exhortait Ryan, chercher cette inspiration dans une vision renouvelée ${ }^{36}$.» Or, comme Ryan puisait son inspiration dans une théologie de l'histoire catholique, il lui paraissait naturel de chercher dans la religion la porte de sortie à la crise que vivait le Canada français. La religion était, à ses yeux, la source première de la civilisation. Dans une pique dirigée à la fois à Duplessis et à ses critiques citélibristes, il rappelait que "contrairement à ce qu'ont pensé les tenants de la philosophie libérale, la religion $[\ldots]$ n'est pas un simple sous-produit ${ }^{37}{ }^{3}$. L'apparition soudaine de multiples critiques du catholicisme et de ses structures vermoulues aurait trahi les aspects religieux de la crise. L'Église institutionnelle n'était plus capable d'adapter suffisamment son apostolat pour répondre aux demandes d'une population de plus en plus urbaine.

Cependant, Ryan croyait qu'il existait, au sein de la société canadiennefrançaise, un hiatus encore plus important. Il observait «le décalage entre la religion et la vie qui s'opère au ras de l'existence quotidienne du petit peuple à la faveur des diverses manifestations d'une civilisation décadente ${ }^{38}$ ». À terme, ce décalage pouvait mener à un état potentiellement morbide de "schizophrénie spirituelle». Les personnes pouvaient en arriver à compartimenter leurs valeurs, continuant à aller à la messe le dimanche et à recevoir les sacrements, sans que les valeurs catholiques aient une quelconque prise réelle sur leur vie, peu importe que ce soit au travail, dans la famille ou dans les loisirs.

Les gens ne commenceront pas d'abord par protester contre la messe du dimanche, ils ne commenceront pas par s'abstenir de toute participation aux cérémonies religieuses et commenceront par trahir sur le plan de la vie personnelle. Sur le plan de la vie la plus intime, là où ils sont convaincus que personne ne s'en apercevra, c'est déjà un signe qu'ils ont commencé à oublier la présence de Dieu dans leurs vies c'est justement ça qu'on appelle la sécularisation de la vie [...] Dans ce qu'on appelle le Canada français ce fait là est déjà profondément entré dans la vie populaire ${ }^{39}$.

C'était là le véritable "drame spirituel». Le style de catholicisme caractéristique de la génération née après 1925 aurait consommé «le divorce

36. BAnQM, FCR, (P558, S12, SS1), Secrétaire national d'Action Catholique, 1995-12-001/360, Claude Ryan, «La Rencontre de deux mondes», Conférence donnée pour la Corporation des Escholiers Griffonneurs, Trois-Rivières, 20 février 1955.

37. Ibid.

38. Ibid.

39. BAnQM, FCR, (P558, S12, SS1), Secrétaire national d'Action Catholique, 1995-12-001/360, Claude Ryan, «Regards sur l'Église du Canada», 30 août 1954. 
prononcé qui sépare la génération aînée de la jeune génération ${ }^{40}$ ». Ryan concédait que les valeurs religieuses de chaque génération, la plus jeune et la plus vieille, n'étaient pas dénuées de pertinence. Cependant, l'atteinte de la maturité sociale et culturelle était menacée par la propagation de certains «mythes» que les groupes entretenaient les uns par rapport aux autres. D’une part, les catholiques plus jeunes jugeaient sévèrement le recours à des doctrines immuables, la diffusion de stéréotypes nationalistes et le maintien d'une religion cléricaliste, et attribuaient une telle sclérose à la génération plus âgée. D’autre part, les catholiques plus âgés considéraient que, en défendant le progrès à tout prix, les plus jeunes en viendraient à consommer la disparition de l'assise religieuse de la société humaine.

Ryan décrivait le catholicisme «traditionnel» auquel aurait souscrit la génération plus âgée dans des termes aussi péjoratifs que ceux qui souscrivaient à la métaphore de la société «folk». Bien sûr, il contestait les conclusions générales des praticiens des sciences sociales comme JeanCharles Falardeau, Fernand Dumont et Guy Rocher, et ce, dans la mesure où il soutenait que la religion canadienne-française était «authentiquement catholique et romaine», et non pas une vague religion primitive "repêchée par le christianisme une forme adultérée ou dégénérée du catholicisme $»^{41}$. Néanmoins, il reste que le jugement de Ryan sur la «tradition» et la génération plus âgée était bien plus dur que celui qu’il portait sur les aspirations de la génération montante. Les lectures qu'il avait faites, très jeune, des écrivains du renouveau catholique français du tournant du siècle, lectures certainement renforcées par ses voyages à travers l'Europe catholique pendant l'été 1952, éclairaient ses penchants idéologiques. Ryan se tenait clairement dans le camp d'un christianisme "pur». Partisan d'une esthétique religieuse moderne et austère, il cherchait à démarquer, parfois agressivement, le christianisme de ses excroissances artistiques baroques et de sa piété post-tridentine. Il considérait d'un oil favorable les nouvelles expériences liturgiques dans les paroisses de France et d'Angleterre, affirmant qu'elles étaient l'« expression vivante d'une Communauté qui n'est qu'un pèlerinage ici-bas et dont la réelle demeure est au ciel $^{42}$ ».

40. BAnQM, FCR, (P558, S12, SS1), Secrétaire national d'Action Catholique, 1995-12-001/360, Claude Ryan, «Rencontre de deux mondes», 20 février 1955.

41. BAnQM, FCR (P558, S12, SS1), Secrétaire national d'Action Catholique, 1995-12-001/361, Claude Ryan, "L'Église catholique et l'évolution spirituelle du Canada français», Chronique sociale de France, novembre 1957.

42. BAnQM, FCR (P558, S10, SS11), 2002-12-003/1, Carnet 41952. 
Pour régler le conflit des générations, qui prenait des proportions alarmantes, il fallait instaurer un climat spirituel "adulte», fondé sur «une aptitude pour la communion». Ces expressions étaient communes chez les intellectuels appartenant au courant personnaliste. Ceux-ci partageaient un sentiment de dégoût pour les pratiques dévotionnelles anciennes et la piété de masse qui obscurcissaient, selon eux, le message chrétien en mettant trop l'accent sur le salut individuel. Il leur semblait qu'il fallait au contraire faire communier le croyant à la vie collective de l’Église ${ }^{43}$. Le message foncier à retenir, c'était que seuls les jeunes gens ayant assimilé le type de spiritualité promu par l'Action catholique avaient une chance d'atteindre la pleine maturité spirituelle. En revanche, la génération plus âgée était destinée à rester figée dans une sorte d'infantilisme religieux tant et aussi longtemps que rien ne serait fait pour remédier à la situation. Pour restaurer la collégialité et le sens du spirituel dans la société canadienne-française, il fallait donc que la génération plus âgée laisse tomber son style nationaliste ringard. Le Canada français devait en finir une fois pour toutes avec l'adéquation entre religion et nation. Plutôt que de se réfugier derrière une espèce de barrière de protection spirituelle, le catholicisme devait s'acheminer vers des valeurs universelles qui ouvrent la société québécoise à des réalités idéologiques et sociales plus larges ${ }^{44}$.

En son point fondamental, l'avenir du lien entre catholicisme et société canadienne-française était déterminé par deux esthétiques de la religion. Il y avait un premier pôle négatif. Ryan le décrivait comme un «catholicisme sociologique». Il comprenait les pratiques sacramentelles, les dévotions populaires, les pèlerinages toujours populaires et la piété religieuse de masse qui était taillée pour assurer le salut individuel. À l'instar de bien d'autres observateurs des années 1950, Ryan assimilait ce régime religieux à la «tradition» et l'interprétait comme un vestige de l’Église post-tridentine. Cependant, il se trompait fort: de telles pratiques religieuses de masse étaient relativement récentes. Elles appartenaient au régime «libéral» et avaient commencé à marquer le paysage religieux du Canada français seulement après $1880^{45}$. Mais peu importe. Vue à travers les lentilles de la

43. BAnQM, FCR (P558, S12, SS1), Secrétaire national d'Action Catholique, 1995-12-001/359, Claude Ryan, «Talk to O.B.T.», 5 septembre 1953.

44. Ibid. Voir aussi Claude Ryan, «Les étudiants s’interrogent: Quel serait pour le chrétien le vrai visage de l'espoir?", Vie étudiante, 21, 8 (mai 1955).

45. Christine Hudon, Prêtres et fidèles dans le diocèse de Saint-Hyacinthe, 1820-1875 (Sillery, Septentrion, 1996); René Hardy, Contrôle social et mutation de la culture religieuse au Québec, 1830-1930 (Montréal, Boréal, 1999); Lucia Ferretti, Entre voisins : la société paroissiale en milieu urbain: Saint-Pierre-Apôtre de Montréal, 1848-1930 (Montréal, Boréal, 1992). 
sociologie et de la psychologie québécoises des années 1950, la «religion sociologique» représentait un amalgame de pratiques folkloriques, de vestiges de vieilles cultures rurales européennes (qui avaient été spontanément traduites, dans un environnement colonial, dans une série de gestes mécaniques de dévotion) et dans la soumission passive à l'autorité d'un clergé local à la fois "embourgeoisé» et intellectuellement médiocre. Le clergé canadien-français, selon Ryan, était déphasé par rapport aux courants culturels modernes. Il n'arrivait pas à sérieusement prendre part aux débats sur la question sociale, la promotion du laïcat et l'internationalisation de la vie humaine ${ }^{46}$.

La persistance d'un tel «catholicisme sociologique» n'était pas seulement une curiosité historique, sans portée nuisible. Pour bien saisir la virulence des propos de Ryan, il faut réaliser que ses inquiétudes allaient au-delà une simple aversion pour des styles religieux dépassés et de mauvais goût. Son discours doit être replacé sur l'horizon intellectuel de l'après-guerre, à un moment où était formulée une conception nouvelle de la sécularisation. Les modèles de l'Europe occidentale et de l'Amérique du Nord étaient à ce moment revêtus d'un prestige inégalé. Pour la première fois, le discours public était dominé par le concept de sécularisation et ce concept définissait les seuls rapports légitimes entre les Églises et les intellectuels. Les membres du clergé, les praticiens des sciences sociales et une grande variété de commentateurs culturels étaient d'accord pour dire que la religion devait être définie en termes sociaux. Dans cette perspective, les institutions religieuses étaient regardées comme des entités passives. Elles n'étaient plus conçues comme des agents dynamiques et modernisateurs.

Les intellectuels s'imaginaient qu'il n'y avait que deux avenirs possibles pour une religion projetée dans le monde moderne. Soit les hauts taux de fréquentation à la messe, l'adhésion aux croyances et aux valeurs catholiques et, par voie de conséquence, l'autorité sociale et culturelle de l'Église seraient dissoute par les acides corrosifs de l'industrialisation et de l'urbanisation. Soit, selon une interprétation plus optimiste, les institutions cléricales s'ouvriraient aux courants plus universels et accepteraient de participer aux grandes transformations sociales (ce qui voulait dire, principalement, assumer leurs responsabilités dans l'élaboration du

46. BAnQM, FCR, (P558, S12, SS1), Secrétaire national d'Action Catholique, 1995-12-001/359, Claude Ryan, "Rapports entre Clergé et laïcs au Canada ", conférence à la Maison Léon XIII Team, 15 février 1954; BAnQM, FCR, (P558, S12, SS1), Secrétaire national d'Action Catholique, 1995-12-001/360, Claude Ryan, «Regards sur l’Église du Canada». 
nouvel ordre démocratique et le développement d'un style de vie contemporain), de telle sorte que la modernisation sociale serait partie prenante de la réalisation du royaume de Dieu sur terre ${ }^{47}$. Cette dernière approche maintiendrait le christianisme à l'avant-garde, certes, mais au prix de laisser la détermination des critères du progrès aux technocrates et aux experts. Pour Ryan, à l'évidence, l'Église était une entité supra-temporelle. En tant qu'Augustinien et lecteur passionné des écrits du cardinal Newman, il doutait de la possibilité de réaliser le royaume de Dieu sur terre. Il demeure que la «nouvelle théologie» faisait s'incarner l’Église dans l'histoire et engageait un dialogue fructueux avec les courants modernes, ce qui légitimait son rôle comme intellectuel.

Les pratiques religieuses suivies par la plupart des catholiques canadiens-français dans les années 1950 étaient néfastes pour deux raisons. D’une part, ces pratiques renforçaient la sécularisation, car elles faisaient passer les gens pour religieux alors que leurs vies étaient en fait divisées en deux : il y avait, d'un côté, une religion publique et, de l'autre côté, un système de relations sociales privées qui ne reflétait plus, dans le quotidien, l'empreinte des valeurs et pratiques chrétiennes. Sur ce point, Ryan s'écartait du cadre conceptuel de plusieurs théoriciens de la sécularisation. Il ne pensait pas que le déclin de la pratique religieuse extérieure reflétait la perte progressive des convictions personnelles et que cette perte suivait forcément le développement de l'industrialisation et de l'urbanisation. Comme l'historien britannique S. J. D. Green l'a si bien dit, l'explication courante de la sécularisation reposait alors sur la notion que "people stop going because they stop believing ${ }^{48}$ ». Les réflexions de Ryan ne gravitaient pas davantage autour de l'axe central des différences de classe. En dépit de ses contacts avec les catholiques sociaux de France, il ne souscrivait pas à l'hypothèse selon laquelle l'adhésion chrétienne devait immanquable-

47. Voir Lucian Hölscher, «Europe in the Age of Secularisation», dans Callum G. Brown et Michael Snape, dir., Secularisation in the Christian World: Essays in Honour of Hugh McLeod (Farnham, Ashgate Publishing, 2010), 197-204.

48. La "thèse de la sécularisation » a été le sujet de maints débats depuis les années 1990: Roy Wallis et Steve Bruce, "Secularization: The Orthodox Model», dans Steve Bruce, dir., Religion and Modernization: Sociologists and Historians Debate the Secularization Thesis (Oxford, Clarendon Press, 1992), 8-30. Un des principaux critiques révisionnistes de la «thèse de la sécularisation» est Callum G. Brown, "The Mechanism of Religious Growth in Urban Societies : British Cities since the Eighteenth Century ", dans Hugh McLeod, dir., European Religion in the Age of Great Cities, 1830-1930 (Londres, Routledge, 1995); Callum Brown, The Death of Christian Britain: Understanding Secularisation 1800-2000 (Londres \& New York, Routledge, 2001; deuxième édition 2009). Pour une présentation juste et équilibrée du débat entre les approches "orthodoxe» et "révisionniste», voir S. J. D. Green, Religion in the Age of Decline: Organisation and Experience in Industrial Yorkshire, 1870-1920 (Cambridge, Cambridge University Press, 1996), chapitre 1. 
ment faiblir dans les zones ouvrières des villes modernes ${ }^{49}$. Vivant dans une société où l'adhésion au catholicisme et à la pratique religieuse était généralisée, il lui était difficile de croire que les différences de classe formaient le vecteur principal de la sécularisation.

Pour lui, le vecteur central de la sécularisation devait être trouvé au sein de l'Église institutionnelle elle-même. En d'autres termes, l'Église n'était pas fondamentalement influencée par des processus extérieurs ${ }^{50}$. L'analyse de Ryan découlait non seulement de son personnalisme augustinien (qui affirmait que l'Église visible contenait ceux destinés au salut comme ceux destinés à la damnation), mais aussi du contexte propre à l'après-guerre. L'Amérique du Nord connaissait alors une période de renaissance religieuse et de "church-building». La "tradition judéo-chrétienne» était auréolée d'un véritable prestige culturel. À l'instar d'une pléiade d'observateurs, qui allaient du sociologue américain Will Herberg, auteur du classique Protestant, Catholic, and Jew (1955), à Pierre Berton, auteur de The Comfortable Pew (1965), Ryan s'inquiétait d'une popularité apparente du christianisme qui aurait masqué, en fait, une participation religieuse superficielle, un conformisme social et une religion faite de confort personnel et de satisfaction individuelle ${ }^{51}$.

Dix ans avant la critique acclamée de Berton (qui fustigera les fidèles canadiens de la classe moyenne), Ryan sonnait l'alarme. Il s'élevait contre le fait que des "principes séculiers» étaient en train de "s'immisci[er] dans la vie de l’Église». Les liens étroits qui s'étaient développés dans l'aprèsguerre entre la religion, le monde des affaires et la banlieue auraient transformé les Églises institutionnelles en simples expressions du matérialisme et du philistinisme ambiants. Les chrétiens seraient devenus des croyants de façade, eux dont la formation théologique aurait été affreusement faible ${ }^{52}$. Derrière la fréquentation massive de la messe et la pratique tout aussi massive des sacrements, Ryan devinait une sécularisation beaucoup plus insidieuse et perfide. Pour lui, la conformité aux prescriptions extérieures de la religion était entièrement compatible avec une croyance personnelle déficiente. Une telle tendance, si elle s'accentuait, pourrait com-

49. Voir BAnQM, FCR, (P558, S12, SS11), 2002-12-003/1, Carnets 1953, 24.11.53.

50. Cette opinion a été défendue avec le plus de conviction par Callum Brown. Celui-ci soutient que le déclin religieux et la déchristianisation ont pour cause la «révolution démographique» qui a eu lieu après les années 1960 .

51. Sur les craintes d'une religion superficielle, voir M. Silk, Spiritual Politics, op. cit., 44-45 ; Nancy Christie, "Belief crucified upon a rooftop antenna": Pierre Berton, The Comfortable Pew, and Dechristianization", dans N. Christie et M. Gauvreau, dir., The Sixties and Beyond... op. cit., 321-350.

52. BAnQM, FCR, (P558, S12, SS1), Secrétaire national d'Action Catholique 1995-12-003/360, Claude Ryan, «Is Canada a Christian Country?», Citizens' Forum, Toronto, 20 décembre 1955. 
promettre l'authenticité de la foi chrétienne et, en dernière analyse, creuser un fossé insurmontable entre l'Église et la culture moderne.

Grand lecteur de Newman, Ryan était convaincu que la vérité religieuse ne pouvait se passer d'une symbiose entre la conscience et l'intellect. Tout autre type de religiosité ne pouvait, selon lui, avoir de valeur spirituelle. Ryan mettait ainsi en contraste le "catholicisme sociologique» avec une spiritualité personnaliste, qui en constituait en quelque sorte le versant positif. Fidèle à la théologie et l'histoire modernes de l'Église, la spiritualité personnaliste favorisait les pratiques liturgiques collectives et appuyait l'idée "moderne» du salut en tant que geste communautaire. Plus fondamentalement, on sentait l'influence grandissante des notions de masculinité et de féminité dans les débats orchestrés par les intellectuels catholiques canadiens-français des années 1950. L’expérience personnelle de Ryan n'était sans doute pas étrangère à de telles préoccupations. Il avait grandi dans un foyer dont le père était absent. L'influence de la mère avait été déterminante sur l'éducation religieuse de ses fils. Enfin, en tant que leader des mouvements d'Action catholique, dont le membership était des deux tiers jusqu'aux trois quarts féminin, il ne pouvait pas ne pas être sensible à la question brûlante du genre ${ }^{53}$.

Écrivant en 1959, Ryan résumait en deux mots le climat culturel catholique: «religion dévirilisée». Il n’hésitait pas à associer les caractéristiques communes du conformisme, de l'autoritarisme, de l'individualisme, de l'habitude et de la routine au fait que le catholicisme tendait à attirer davantage des femmes que des hommes ${ }^{54}$. Pour lui, le catholicisme exprimait des émotions marginales et artificielles, une affirmation qui se voulait une référence claire à l'influence trop prépondérante des femmes. Ryan ne remettait pas en question la sincérité d'un tel catholicisme, du moins publiquement, mais il pensait qu' «il n'était ni viril ni adulte ${ }^{55}$ ». En effet, le "catholicisme sociologique» typique du Canada français était d'abord l'expression d'une culture de masse nord-américaine, à laquelle un large groupe de praticiens des sciences sociales et d'observateurs des deux côtés de l'Atlantique avait déjà attribué des caractéristiques "féminines». La hiérarchie culturelle de l'époque était très structurée par le genre. Faut-il s'étonner que ce système de valeurs ait exalté le rôle des intellectuels

53. Sur la très forte proportion de femmes dans les divers mouvements d'action catholique spécialisée, voir Jean Hamelin, Histoire du catholicisme québécois, Tome 2 : de 1940 à nos jours (Montréal, Boréal, 1984), 127.

54. Claude Ryan, Esprits durs, cœurs doux: La vie intellectuelle des militants chrétiens (Montréal, Action Catholique Canadienne, 1959), 11.

55. SAUM, FACC, P16, B6, 3,18, Claude Ryan, «Les laïcs et la vie liturgique au Canada», avril 1954. 
masculins comme arbitres culturels? Paradoxalement, la plupart de ces hommes se considéraient comme des libéraux, voire même des radicaux, mais ils restaient prisonniers d'un puissant conservatisme lorsqu' il s'agissait de défendre les frontières culturelles de leur univers ${ }^{56}$. Il y a peu de doute que Ryan partageait l'essentiel de leurs opinions.

Ryan voulait se présenter comme «homme d'action» et "travailleur intellectuel», ce qui traduisait la tentative plus générale des intellectuels de l'Amérique du Nord d'affirmer la virilité des entreprises culturelles des membres de l'élite ${ }^{57}$. Une des principales critiques du «catholicisme sociologique» formulées par Ryan était justement que ce catholicisme dévirilisait la culture canadienne-française. La promotion d'une piété de masse encourageait la construction de la religion comme espace féminin, avec pour résultat que les hommes ne pouvaient s'y sentir à l'aise. En faisant reposer de manière exagérée les prétentions sociales et culturelles du catholicisme sur les structures cléricales, on dévaluait la vie essentielle de l'esprit. On se trouvait par conséquent dans l'incapacité de parler aux élites montantes de la classe moyenne du Canada français. Ces "élites vivantes» se logeaient dans ce que l'analyse contemporaine allait appeler le «domaine du savoir»: enseignants des écoles secondaires, professeurs d'université, journalistes, artistes, vulgarisateurs et directeurs des mouvements d'Action catholique ${ }^{58}$.

Les mentalités religieuses définies par le genre posaient une menace à la fois à l'avenir du catholicisme, comme dimension centrale de la société canadienne-française, et à l'achèvement de la démocratie moderne au Québec. Des dévotions particulières, des pratiques habitudinaires et le conformisme - les traits "féminins» du catholicisme - n'étaient pas en soi mauvais. Mais, quand ils se retrouvaient au centre de la religion, comme c'était le cas dans le catholicisme «traditionnel» québécois, ils avaient une propension malheureuse à conduire à la dégénération culturelle. Convaincu de la justesse de ce sombre pronostic, Ryan voulait que ses compatriotes en finissent avec la prédominance de l'élément féminin. À défaut de quoi, l'élément féminin finirait par provoquer l'évacuation de la religion de la sphère publique, car l'élite la plus énergique et la plus active - lire la plus masculine - en viendrait à regarder la religion comme

56. J. Gilbert, Men in the Middle, op. cit., 189-214.

57. BAnQM, FCR, (P558, S12, SS11), Secrétaire national d'Action Catholique, 1995-12-001/361, Claude Ryan, "Crise de conscience des intellectuels canadiens-français», 2 mars 1956.

58. BAnQM, FCR, (P558, S12, SS1), Secrétaire national d'Action Catholique, 1995-12-001/359, Claude Ryan, «Rapports entre Clergé et laïcs au Canada». 
quelque chose d'obsolète et d'inutile ${ }^{59}$. Un tel dénouement ramènerait le catholicisme vers le terrain de la "tradition", ce qui empêcherait la jonction souhaitée entre les éléments dynamiques de la religion et les forces de la démocratie politique et sociale qui auraient pu, espérait Ryan, maintenir le catholicisme au cour de la vie canadienne-française.

Les expressions de féminité et de masculinité qui caractérisaient la mentalité religieuse auraient constitué, dès lors, le principal vecteur de sécularisation dans le Québec des années 1950. Il n’est pas banal de noter que le discours de Ryan anticipait l'analyse de Callum Brown. Dans son important ouvrage The Death of Christian Britain, Brown attribue l'effondrement catastrophique, dans les années 1960, des cadres religieux traditionnels à la décision des femmes de quitter les institutions chrétiennes ${ }^{60}$. Pour Ryan, toutefois, le problème du déclin religieux était essentiellement masculin. Il était causé par le dégoût que les hommes professionnels et militants éprouvaient pour un catholicisme de masse féminin et conformiste.

Il est difficile de ne pas conclure que, au milieu des années 1950, Ryan considérait la "crise religieuse», dans sa forme la plus exacerbée, comme une expression des tensions qui se seraient développées entre l’Église et une nouvelle classe d'hommes intellectuels. Au début de l'année 1956, il décrivait comment, durant les derniers quinze ans, il avait suivi la naissance et la reconnaissance progressive d'une nouvelle génération d'intellectuels. Ces hommes se démarquaient à la fois par leur compétence et un sens plus fort de la quête spirituelle: ils possédaient un «esprit scientifique» et se démarquaient par «le souci de dépasser le régionalisme pour communier à la culture universelle $»^{61}$. Dans l'esprit de Ryan, ce constat signifiait que la plupart de ces intellectuels avaient abandonné la synthèse traditionnelle du nationalisme et du catholicisme ethnique qui avait caractérisé la période de l'entre-deux-guerres. Il s'agissait désormais pour eux de mettre de l'avant les valeurs universelles de la civilisation occidentale.

Ryan notait que la vaste majorité des intellectuels canadiens-français se considéraient comme catholiques. Néanmoins, l’Église ne devait pas tirer de ce fait quelque source de fierté. Reprenant un diagnostic psychanalytique à la mode dans l'après-guerre, Ryan jugeait que les membres de sa génération, maintenant dans la trentaine et la quarantaine, avaient atteint le stade

59. Claude Ryan, «La religion des canadiens-français», Cahiers d'Action catholique, 172 (février 1955): 244. Voir aussi SAUM, FACC, P16/B6, 3,18, "Les laïcs et la vie liturgique au Canada».

60. C. Brown, The Death of Christian Britain... op. cit., dernier chapitre.

61. BAnQM, FCR (P558,S12, SS1),Secrétaire national d'Action catholique, Claude Ryan, « Rapports entre clergé et laïcs au Canada» 
de l'adolescence. À cet âge, les tensions entre l'esprit universaliste et scientifique et les valeurs catholiques devenaient plus sensibles. Vue dans cette perspective, la psyché collective des intellectuels pouvait être résumée par "un état de malaise fort répandu; ce malaise porte, non pas tout sur Dieu, mais sur l'Église ${ }^{62} »$. Cependant, Ryan était prudent de ne pas nommer ce malaise une "crise de la culture", même si la culture et la religion se chevauchaient énormément, le catholicisme étant une dimension intégrale et même centrale de l'identité canadienne-française ${ }^{63}$.

\section{CONCLUSION}

Dans les années 1950, Ryan gardait espoir. Il croyait que les intellectuels canadiens-français allaient résoudre leur crise d'adolescence et pourraient négocier une transition vers l'âge adulte, d'abord pour eux-mêmes comme individus et comme intellectuels, et ensuite pour leur société tout entière. L'âge adulte représentait, pour Ryan, un état qui, tout en maintenant la primauté du catholicisme dans la vie culturelle, rendrait la religion plus compétente et plus rationnelle, et affirmerait la suprématie des valeurs humaines universelles. Ryan pensait qu'il n'était pas opportun, en temps de crise, de faire des généralisations alarmistes ou pessimistes. C'était plutôt le temps, pour les individus et les sociétés, de poser des «questions de FOND $»^{64}$.

$\mathrm{Au}$ dire de Ryan, l'atteinte de l'âge adulte exigeait l'intégration des croyances catholiques (définies non pas à partir d'un degré de piété ou d'enthousiasme religieux, ou en fonction d'institutions nouvelles créées dans le but de compenser les lacunes des sociétés humaines, mais comme une synthèse de l'enseignement théologique et de la conscience) et les valeurs modernes de la nouvelle communauté intellectuelle. Pareille intégration permettrait - du moins Ryan l'espérait-il - de redonner aux intellectuels masculins leur pertinence et d'en finir avec les divisions de plus en plus acrimonieuses dans lesquelles ils étaient empêtrés. Son objectif plus ambitieux était de refaire le lien entre l'Église et les intellectuels. Il cherchait un élément culturel unifiant qui synthétiserait, dans un constant dialogue, les valeurs venues du passé et celles de l'avenir, et ainsi

62. BAnQM, FCR (P558, S12, SS1), Secrétaire national d'Action Catholique, Claude Ryan, «Rapports entre Clergé et laïcs au Canada ".

63. BAnQM, FCR, (P558, S12, SS1), Secrétaire national d'Action Catholique, 1995-12-001/363, Claude Ryan, «Les collèges classiques et la formation spirituelle et religieuse des professionnels Canadiensfrançais», Université Laval, Faculté des Arts, Commission du programme, 24 avril 1958.

64. BAnQM, FCR (P558, S12, SS11), Carnets 1958, «Are French-Canadians Christian?» : «questions de FOND». 
servirait à redonnerer à l'identité canadienne-française son unité. On retrouve ici l'impératif augustinien qui demande de chercher un accord fondamental à travers un processus continu de communication et d'échange. Seulement ainsi le Canada français pouvait-il aspirer à fonder une civilisation et éviter les conséquences débilitantes d'une "universalisation par en-bas» qui étaient en train de miner les autres sociétés occidentales aux prises avec le cauchemar de la culture de masse ${ }^{65}$. L'incapacité à réaliser un tel objectif condamnerait l'homme canadien-français à la

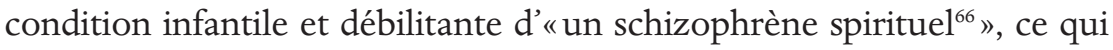
serait le prélude à la déchristianisation complète de sa société et sa mort culturelle.

Texte traduit par Jean-Philippe Warren relu et approuvé par l'auteur 